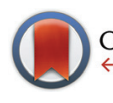

CrossMark $\leftarrow$ click for updates

Cite this: Dalton Trans., 2015, 44 5032

Received 23rd September 2014, Accepted 3rd February 2015

DOI: $10.1039 /$ c4dt02916j

www.rsc.org/dalton

\title{
Characterisation and application of ultra-high spin clusters as magnetic resonance relaxation agents
}

\author{
Gisela Guthausen, ${ }^{\star a}$ Julyana R. Machado, ${ }^{a}$ Burkhard Luy, ${ }^{a}$ Amer Baniodeh, b,c \\ Annie K. Powell, ${ }^{b, c}$ Steffen Krämer, ${ }^{d}$ Florian Ranzinger, ${ }^{a, e}$ Maria P. Herrling, ${ }^{e}$ \\ Susanne Lackner ${ }^{\mathrm{e}}$ and Harald Horn ${ }^{\mathrm{e}}$
}

\begin{abstract}
In Magnetic Resonance Tomography (MRT) image contrast can be improved by adding paramagnetic relaxation agents such as lanthanide ions. Here we report on the use of highly paramagnetic isostructural

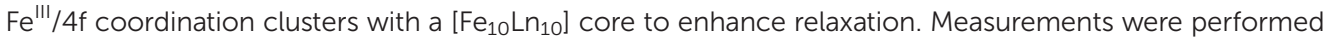
over the range of ${ }^{1} \mathrm{H}$ Larmor frequencies of $10 \mathrm{MHz}$ to $1.4 \mathrm{GHz}$ in order to determine the relevant parameters for longitudinal and transverse relaxivities. Variation of the lanthanide ion allows differentiation of relaxation contributions from electronic states and molecular dynamics. We find that the transverse relaxivities increase with field, whereas the longitudinal relaxivities depend on the nature of the lanthanide. In addition, the $\mathrm{Gd}^{\prime \prime \prime}$ analogue was selected in particular to test the interaction with tissue observed using MRT. Studies on biofilms used in waste water treatment reveal that the behaviour of the high-spin clusters is different from what is observed for common relaxation agents with respect to the penetration into the biofilms. The $\mathrm{Fe}_{10} \mathrm{Gd}_{10}$ cluster adheres to the surface of the biofilm better than the commercial agent Gadovist.
\end{abstract}

\section{Introduction}

In MRT and also in NMR spectroscopy, paramagnetic molecules are of special importance regarding their relaxation behaviour in solution (paramagnetic relaxation enhancement, PRE) and their influence on line shifts (pseudocontact shift, PCS). In the present context, PRE is examined for newly available highly paramagnetic coordination clusters with up to 120 unpaired electrons. These $3 \mathrm{~d} / 4 \mathrm{f}$ clusters form cyclic structures which - to the best of our knowledge - are unique in the category of potential relaxation agents. The high spin clusters $\left[\mathrm{Fe}^{\mathrm{III}}{ }_{10} \mathrm{Ln}^{\mathrm{III}}{ }_{10}(\mathrm{Me}-\mathrm{tea})_{10}(\mathrm{Me}-\mathrm{teaH})_{10}\left(\mathrm{NO}_{3}\right)_{10}\right]$ are abbreviated as $\mathrm{Fe}_{10} \mathrm{Ln}_{10}$. At room temperature they have magnetic moments corresponding to the sum of the contributions of the isolated ions. ${ }^{1}$ The clusters were synthesized as outlined recently. ${ }^{2}$ The $\mathrm{Fe}_{10} \mathrm{Ln}_{10}$ compounds are soluble in water ${ }^{3}$ and other solvents.

\footnotetext{
${ }^{a}$ Pro ${ }^{2}$ NMR at the Institutes for Biological Interfaces and for Mechanical Processes Engineering and Mechanics, KIT, Adenauerring 20b, D-76131 Karlsruhe, Germany. E-mail: gisela.guthausen@kit.edu

${ }^{b}$ Institute of Nanotechnology, KIT, Hermann-von-Helmholtz-Platz 1, 76344 Eggenstein-Leopoldshafen, Germany

${ }^{c}$ Institute of Inorganic Chemistry, KIT, Engesserstr. 15, D-76131 Karlsruhe, Germany ${ }^{d}$ Laboratoire National des Champs Magnétiques Intenses, LNCMI-CNRS (UPR3228), UJF, UPS, and INSA, 25 rue des Martyrs, BP 166, 38042 Grenoble, France ${ }^{e}$ EBI, Chair of Water Chemistry and Water Technology, KIT, Engler-Bunte-Ring 1, D-76131 Karlsruhe, Germany
}

Apart from these cyclic coordination clusters there are very few other examples of such $\mathrm{d} / \mathrm{f}$ systems. ${ }^{4}$

In addition to the large number of unpaired electrons in the $\mathrm{Fe}_{10} \mathrm{Ln}_{10}$ systems, the molecular tumbling in solution deviates from the usual behaviour of small molecules such as single ion gadolinium complexes. ${ }^{3}$ These points are of fundamental interest since these molecules have nanoscale dimensions and are thus expected to behave differently from both single-centre coordination compounds and nanoparticles used as contrast agents. Thus, the first step is to provide a suitable database in order to develop a theoretical model which describes the clusters' properties with respect to PRE. The series of compounds available makes it possible to investigate the individual electronic contributions to the hyperfine relaxation and the parameters that depend on the molecular environment by varying the lanthanide ion and allow us to discover the influence of the time-modulated hyperfine couplings.

From the application point of view of PRE in magnetic resonance imaging or tomography (MRT), generating a contrast enhancement is required. This is especially relevant in cases where the difference in chemical composition is small making structures hard to distinguish. Potential relaxation agents are of interest regardless of whether the application is in medical diagnostics or related to material science. PRE reduces both the longitudinal relaxation times $T_{1}$ as well as the transverse relaxation times $T_{2}$, which lead to different effects on image contrast. On one hand, the reduction of $T_{1}$ is equivalent with a 
faster magnetization build-up. As in MRT measurements are most often performed in dynamic magnetization equilibrium, this leads to larger signal amplitudes in the presence of relaxation agents. PRE of $T_{1}$ is therefore often called a positive contrast enhancement. On the other hand, the reduction of the transverse relaxation time leads to a reduction of signal amplitude. MRT in material science often utilizes spin echo sequences, which involve an echo time with a lower limiting value. Therefore, reducing $T_{2}$ leads to a reduction of signal in the presence of paramagnetic moieties. The knowledge of the clusters relaxation properties gives a first indication, which contrast can be expected in MRT.

This approach has the limitation that the paramagnetic molecules must be in solution. An intriguing question, especially in the present case of relatively large clusters, is how they behave in inhomogeneous systems. Apart from clustering and aggregation, tumbling, diffusion and exchange rates depend on the chemical environment as quantified in the equations describing classical PRE. ${ }^{5}$ When imaging heterogeneous systems the PRE's of the components have to be known, if the concentrations of the paramagnetic moieties are to be determined quantitatively. In the present study on biofilms, a fundamental question is the degree of mass transfer of compounds into the biofilm matrix. Relaxation agents exhibiting different chemical surface properties and sizes can be used as probes with the prerequisite that this effect still occurs when the moieties are within the biofilm structure.

\section{Experimental}

\section{Sample preparation}

Sample preparation of the aqueous solutions of $\mathrm{Fe}_{10} \mathrm{Ln}_{10}$. The general synthetic strategy is a one-pot reaction where the lanthanide and iron nitrate salts reacted with the racemicligand in a mixture of solvents after addition of base to yield the $\mathrm{Fe}_{10} \mathrm{Ln}_{10}$ coordination cluster systems as extremely pale yellow material. The general synthetic details as well as the role of the ligand-chirality in the stability of the structure molecules are described in ref. 2, 6. Several tests were performed concerning the stability of the cluster in water or other solvents such as steady-state absorption spectroscopy. The clusters are found to be stable and do not decompose into their components. ${ }^{6}$ In the crystal structures the lattice solvent molecules (acetonitriles) are remote from the metal ions.

The five lanthanides clusters $\mathrm{Fe}_{10} \mathrm{Dy}_{10}, \mathrm{Fe}_{10} \mathrm{Er}_{10}, \mathrm{Fe}_{10} \mathrm{Gd}_{10}$, $\mathrm{Fe}_{10} \mathrm{~Tb}_{10}$ and $\mathrm{Fe}_{10} \mathrm{Tm}_{10}$ with a concentration of $1 \mathrm{mM}$ in water were selected for the relaxivity studies. They were diluted using $\mathrm{D}_{2} \mathrm{O}(90 \%)$ and $\mathrm{H}_{2} \mathrm{O}(10 \%)$ to avoid radiation damping, especially at high magnetic fields. The concentrations investigated in the NMR experiments were: $0.05 \mathrm{mM}, 0.10 \mathrm{mM}$, $0.20 \mathrm{mM}, 0.30 \mathrm{mM}, 0.40 \mathrm{mM}, 0.50 \mathrm{mM}, 0.60 \mathrm{mM}, 0.80 \mathrm{mM}$ and $1 \mathrm{mM}$. In the ultra-high fields $>600 \mathrm{MHz}$, only concentrations of interest were selected which were $0.30 \mathrm{mM}$, $0.60 \mathrm{mM}, 0.80 \mathrm{mM}$ and $1 \mathrm{mM}$ due to the short time period of access to the magnet. Measurements at ${ }^{1} \mathrm{H}$-Larmor frequencies from $10 \mathrm{MHz}$ to $600 \mathrm{MHz}$ were performed on samples in Wilmad sample tubes (OD $5.0 \mathrm{~mm}$ ). At higher fields above ${ }^{1} \mathrm{H}$-Larmor frequencies of $600 \mathrm{MHz}$, Wilmad EPR capillary tubes of OD $1.2 \mathrm{~mm}$ and ID $1.0 \mathrm{~mm}$ with a length of $12 \mathrm{~mm}$ were used. The capillaries borders were closed with proton free fluorinated polymer grease (DAIFLOIL, Daikin, China). By Teflon ribbon, the capillaries were fixed in the horizontally oriented NMR coil. Further details are summarized in ref. 3.

Cultivation and preparation of the biofilm carriers for MRT. A laboratory scale moving bed biofilm reactor $(V=1 \mathrm{~L})$ was operated for 90 days with $\mathrm{K} 1$ plastic carrier material (AnoxKaldnes $\mathrm{AB}$, Sweden) at a filling ratio of $25 \%$. The carrier material has a cylindrical shape with four open sections and has a specific surface area of $500 \mathrm{~m}^{2} \mathrm{~m}^{-3}$. The reactor was continuously fed with acetate $\left(1200 \mathrm{mg} \mathrm{L}^{-1}\right)$ as main substrate to grow the heterotrophic biofilms. The reactor was continuously aerated with pressurized air to ensure mixing and oxygen saturation. The hydraulic retention time was approximately 0.2 days. For the MRT experiments, the $\mathrm{K} 1$ carrier was rinsed with tap water to remove remaining substrate and particulate matter before the installation into the sample holder. After measuring without relaxation agents, and injection of either 4Hydroxy-TEMPO (Sigma-Aldrich Chemie $\mathrm{GmbH}$ ), the $\mathrm{Fe}_{10} \mathrm{Gd}_{10}$ cluster or Gadovist (Gd-DO3A-butrol6, Bayer HealthCare AG, Leverkusen, Germany) was performed. The injection concentrations were in $\mathrm{mM}$ range, but the relaxation agents were diluted immediately due to the fact that the carrier was placed completely in water.

\section{NMR spectrometers and pulse sequences}

In the relaxivity study, several NMR systems were used in order to cover the interesting Larmor frequency range from $10 \mathrm{MHz}$ to $1.4 \mathrm{GHz}$. The MRT images were obtained at $200 \mathrm{MHz}$ applying different parameter schemes to obtain a dedicated contrast.

${ }^{1} \mathrm{H}$-frequencies ${ }^{10-600} \mathrm{MHz}$ for relaxivity measurements and MRT. Instruments of the "Bruker the minispec" series were utilized for investigating $T_{1}$ and $T_{2}$ relaxation at ${ }^{1} \mathrm{H}$-Larmor frequencies of 10, 20 and $60 \mathrm{MHz}$. $T_{1}$ was measured by the saturation recovery pulse sequence. $T_{2}$ was determined from transverse magnetization decays recorded by a CPMG multi-echo sequence while recording every echo, which is facilitated by the low magnetic field homogeneity of the TD-NMR instruments.

On a Bruker $400 \mathrm{MHz}$ WB spectrometer, a SEF $400 \mathrm{MHz}$ W1 probe for $5 \mathrm{~mm}$ sample tubes were used; at $600 \mathrm{MHz}$, a TBI probe for $5 \mathrm{~mm}$ sample tubes with actively shielded $z$-gradients was placed into a $600 \mathrm{MHz}$ SB magnet. The data acquisition software varied from XWINNMR $3.5(600 \mathrm{MHz})$ to TOPSPIN1.5 $(200 \mathrm{MHz})$ to TOPSPIN2.1 (400 MHz). $T_{1}$ was obtained from mono-exponential fits on data from inversion recovery; $T_{2}$ resulted from single echo CPMG measurements as implemented on Bruker high field NMR spectrometers. Data were processed within TOPSPIN. All samples were measured at room temperature. Typical sequence parameters of the measurements are summarized in ref. 3. 
A Bruker $200 \mathrm{MHz}$ SWB instrument was used for relaxivity measurements, being equipped with a Diff30 probe for $5 \mathrm{~mm}$ sample tubes. For imaging, the multi-slice multi-echo sequence was applied on a micro2.5 set-up with a $25 \mathrm{~mm}$ birdcage resonator. Parameters were adjusted such that either dominantly $R_{1}$ or $R_{2}$ contrast was achieved. Carrier matierials with attached cultivated bioilm were measured first without any relaxation agents, then a time series of images was acquired to detect a possible permeation of the paramagnetic moieties into the biofilm. The contrast parameter for the images were repetition times and echo time, which were chosen to $T_{\mathrm{R}}=800 \mathrm{~ms}$ and $\tau_{\mathrm{E}}=4.5 \mathrm{~ms}$ for $T_{1}$ weighting, $T_{\mathrm{R}}=10 \mathrm{~s}$ and $\tau_{\mathrm{E}}=50 \mathrm{~ms}$ for $T_{2}$ weighting, and $T_{\mathrm{R}}=10 \mathrm{~s}$ and $\tau_{\mathrm{E}}=3.2 \mathrm{~ms}$ for PD images.

${ }^{\mathbf{1}} \mathrm{H}$-frequencies 850-1400 $\mathrm{MHz}$ for relaxivity measurements. The Laboratoire National des Champs Magnétiques Intenses (LNCMI) in Grenoble disposes a 24 MW resistive magnet providing variable fields up to $35 \mathrm{~T}$ in a $34 \mathrm{~mm}$ room temperature bore and home-built NMR hardware allowing variable frequency NMR up to 1.4 GHz. Despite of the low magnetic field homogeneity in the order of $10 \mathrm{ppm}$ over $1 \mathrm{~mm}$ and despite of field fluctuations in the range of $10 \mathrm{ppm},{ }^{1} \mathrm{H}$-relaxation measurement were still feasible, using small sample volumes in order to reduce spatially induced field inhomogeneity. Furthermore single shots were recorded. Carr-Purcell-MeiboomGill (CPMG) echo trains could cope with the field fluctuations satisfactorily. Cylindrical sample tubes with $1.2 \mathrm{~mm}$ outer diameter, filled with the liquid sample up to a height of about $3 \mathrm{~mm}$ at most, were placed in a specially designed single-resonance ${ }^{1} \mathrm{H}$-NMR probe enabling in situ tuning of NMR frequencies between $850 \mathrm{MHz}$ and $1.4 \mathrm{GHz}$. Temperature is kept constant at $300 \mathrm{~K}$ within $1 \mathrm{~K}$ by an active temperature stabilization system. FIDs after an inversion and subsequent recovery as well as CPMG echo trains were recorded to determine the longitudinal and the transverse relaxation rates $R_{1}$ and $R_{2}$, respectively, as a function of magnetic field. ${ }^{3}$

\section{Results and discussion}

\section{Relaxation study of $\mathrm{Fe}_{10} \mathrm{Ln}_{10}$ clusters}

From the perspective of MRT, the paramagnetic clusters $\mathrm{Fe}_{10} \mathrm{Ln}_{10}$ (Fig. 1) are an interesting substance class because of their magnetic properties. Due to the hyperfine coupling between electron spins on the cluster and nuclei in the neighbourhood together with field fluctuations due to electron spin relaxation, molecular tumbling, diffusion, or chemical exchange, the relaxation properties of molecules surrounding the cluster will change. In the case of conventional contrast agents these mechanisms are well understood ${ }^{5,7}$ and can be calculated analytically, provided that the parameters of the multi-variable equations are known. ${ }^{5}$ Often limiting cases of this rather complex theoretical framework for PRE are discussed. ${ }^{5}$ In the present paper, we explored a different way for the characterization of the molecular clusters by variation of the lanthanide ions also mentioned in ref. 8. The molecular architecture allows the synthesis of a series of $\mathrm{Fe}_{10} \mathrm{Ln}_{10}$ clusters

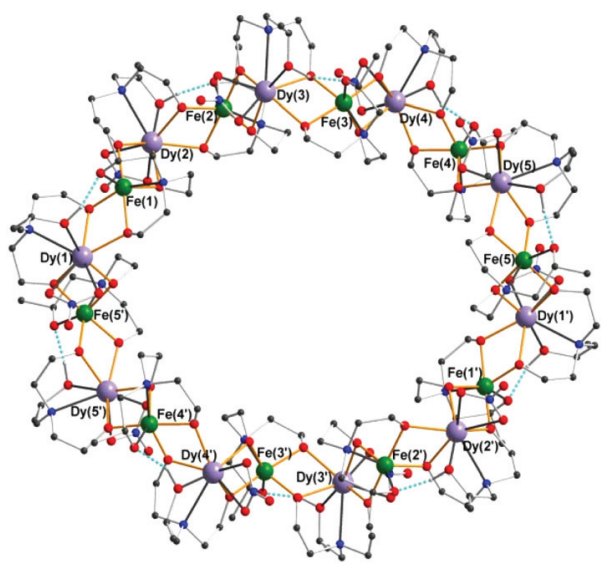

Fig. 1 Structure of the clusters on the example of $\mathrm{Fe}_{10} \mathrm{Dy}_{10}$ : The paramagnetic ions form a ring, which is surrounded by Me-tea units. The long axes of the elliptical cluster amount to 2.84 and $2.63 \mathrm{~nm}$; the height is $1.27 \mathrm{~nm}$. The inner cavity has dimensions of 1.22 and $0.83 \mathrm{~nm}$, respectively.

with different electronic properties and therefore hyperfine couplings. In terms of NMR relaxation, the contributions to PRE are varied due to different electronic relaxation contributions. This contribution to PRE is characterized by the hyperfine coupling and the electronic correlation times and can therefore be differentiated from the contributions of molecular tumbling, diffusion and chemical exchange. In the present study, the investigated lanthanides within the $\mathrm{Fe}_{10} \operatorname{Ln}_{10}$ clusters are $\mathrm{Gd}^{\mathrm{III}}$ (total angular momentum quantum number $j=7 / 2), \operatorname{Tb}^{\mathrm{III}}(j=6), \mathrm{Dy}^{\mathrm{III}}(j=15 / 2), \mathrm{Er}^{\mathrm{III}}(j=15 / 2)$, and $\mathrm{Tm}^{\mathrm{III}}$ $(j=6)$.

The relaxivities (Fig. 2) were obtained by measuring the longitudinal and the transverse relaxation rates for different contrast agent concentrations over the range of ${ }^{1} \mathrm{H}$ Larmor frequencies of $10 \mathrm{MHz}$ to about $1.4 \mathrm{GHz}$. The relaxation rates were obtained by monoexponential fits on the experimental data. These relaxation rates are expected to linearly depend on concentration, which was indeed observed in analogy to ref. 3. A linear fit was performed on the relaxation rates as a function of concentration. The resulting slopes represent the relaxivities (Fig. 2). The concentration, which entered the relaxivity, is based on the whole cluster rather than on the single paramagnetic ion. We currently prefer this presentation, as little is known whether the relaxation can be understood regarding the whole complex as a single magnetic moment or regarding each single ion in the cluster in terms of a conventional molecular relaxation agent. In addition, one should keep in mind that the dimension of a single water molecule is rather small compared to the cluster size such that the question whether the cluster acts as a single moiety or as a sum of individual ions is not trivial. The answer will certainly depend also on the degree and nature of coupling of the electronic $d$ and f orbitals of the ions via the nearby p orbitals of the bridging oxo-groups from the ligands.

At low magnetic fields the longitudinal relaxivity $r_{1}$ shows a quite interesting behaviour as a function of the Ln ions: 

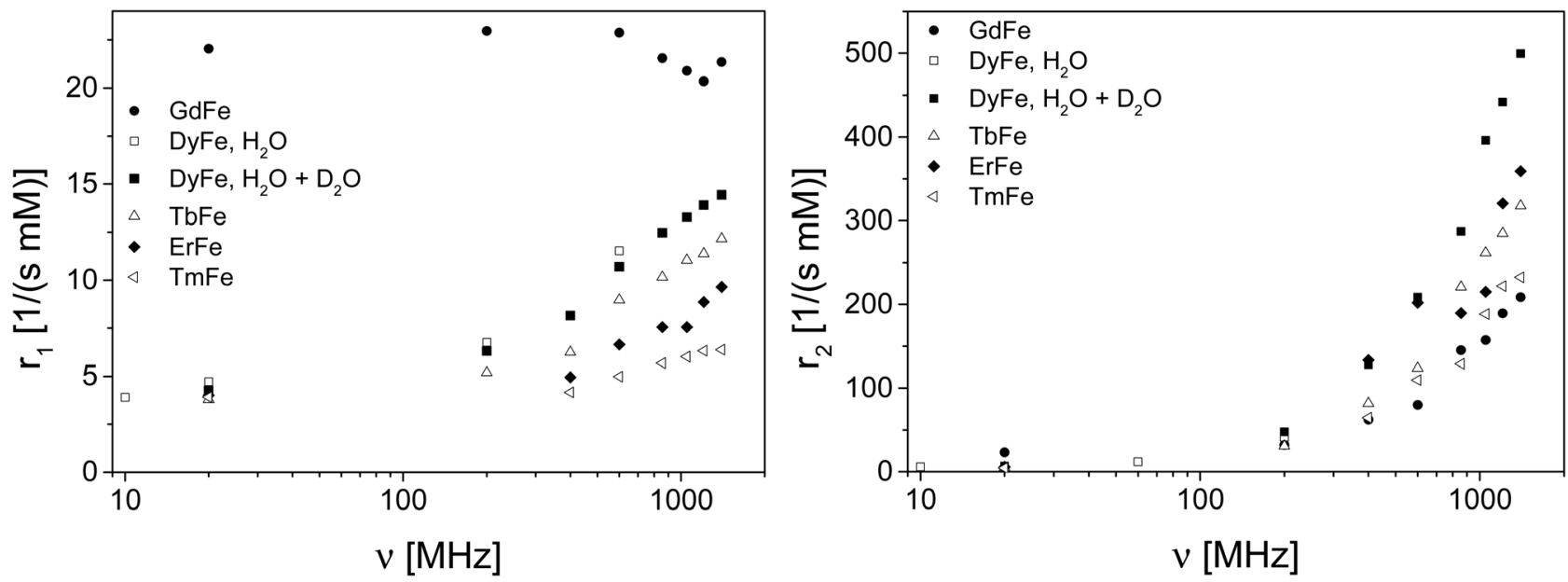

Fig. 2 Longitudinal $\left(r_{1}\right)$ and transverse $\left(r_{2}\right)$ relaxivities of water in the presence of the diverse $\mathrm{Fe}_{10} \mathrm{Ln}_{10}$ clusters. Except of Fe $\mathrm{Fe}_{10} \mathrm{Gd}_{10}$ the complexes show a pronounced increase of $r_{1}$ and $r_{2}$ relaxivities with magnetic field and are therefore potential relaxation agents especially in high field MRT.

Except of the Gd cluster, all contrast agents show moderate relaxivities despite of the large number of unpaired electrons, which is expected for outer sphere relaxation. Furthermore, $r_{1}$ increases more or less pronounced with field. This behaviour differs from that of common relaxation agents: Often, the relaxivity decreases with increasing field, ${ }^{5,9-12}$ already at moderate field strengths. We observed a steady increase of relaxivity up to the highest currently available magnetic fields in a similar way as it was observed at lower fields also for Ln ions in aqueous solutions, ${ }^{13,14} \mathrm{Ni}^{\mathrm{II}}$ complexes ${ }^{15}$ and magnetic nanoparticles. ${ }^{16}$ Further measurements on $\mathrm{Fe}_{10} \mathrm{Lu}_{10}$ in the range $10 \mathrm{MHz}$ to $600 \mathrm{MHz}$ do not show the typical increase of $r_{1}$ and $r_{2}$ as a function of ${ }^{1} \mathrm{H}$ Larmor frequency, leading to the conclusion that this observation can be attributed to the paramagnetism of the $\mathrm{Ln}^{\mathrm{III}}$ ions in the clusters. An additional hint on the importance of the Ln electronic states and their correlations times is given by the PRE of the $\mathrm{Fe}_{10} \mathrm{Gd}_{10}$ cluster (Fig. 2) in comparison to the other $\mathrm{Fe}_{10} \mathrm{Ln}_{10}$ moieties: $r_{1}$ $\left(\mathrm{Fe}_{10} \mathrm{Gd}_{10}\right)$ behaves approximately field independent at moderate fields, except in the very high field limit where a small decrease is observed.

In order to qualitatively explain the observed relaxivity (Fig. 2) different determining factors should be considered: molecular tumbling rates, diffusion, chemical exchange as well as field dependence and dynamics of the electron spin states. As far as the cluster is currently analysed with respect to its physical properties, the dominant part of PRE is attributed to outer sphere relaxation, as no direct binding site for water molecules is available at the $\mathrm{Fe}^{\mathrm{III}}$ and $\mathrm{Ln}^{\mathrm{III}}$ ions. Dipolar and Curie contributions are of major importance for PRE of diverse Ln contrast agents, which is supported by experimental investigations. ${ }^{13,14}$ However, inner sphere contributions are significant in these investigations, which is not the case for the $\mathrm{Fe}_{10} \mathrm{Ln}_{10}$ clusters.

The known physical parameters of Ln ions are summarized with respect to PRE in literature: ${ }^{13,17}$ In particular, the electronic correlation times and the anisotropy parameters of the hyperfine coupling differ across the lanthanide series. An effective radius for PRE is assigned for the different lanthanides. ${ }^{17}$ When comparing the findings in ref. 13, 14,17 with Fig. 2, a qualitative correlation can be established, although without taking into account the role of the $\mathrm{Fe}^{\mathrm{III}}$ ions. An especially interesting case is the cluster $\mathrm{Fe}_{10} \mathrm{Gd}_{10}$ : The electronic correlation time of $\mathrm{Gd}^{3+}$ spin states is usually rather long, the hyperfine anisotropy is negligible, and the effective radius for PRE is rather large. These exceptional properties are reflected in Fig. 2. The large number of paramagnetic ions leads to a spread of the electronic energy levels, which is confirmed by preliminary EPR measurements at room temperature. In this case relaxation theory for a single centre is not adequate $a$ priori. On the other hand, the observed PREs differ also from typical observations in superparamagnetic clusters and nanoparticles, ${ }^{11,18,19}$ therefore the models for the description of PRE of these moieties cannot be applied directly to the data in Fig. 2. Magnetically coupled systems such as the present $\mathrm{Fe}_{10} \mathrm{Ln}_{10}$ clusters do not necessarily follow the standard approaches to interpreting PRE. ${ }^{5}$

In summary, PRE is influenced by a variety of molecular parameters such as diffusional and rotational correlation times. The electronic correlation times, the symmetry of the hyperfine couplings and the susceptibility of the $\mathrm{Ln}^{\text {III }}$ ions differ by orders of magnitude, which are important parameters in PRE. Furthermore, polarization effects in the high spin clusters can occur at the rather high magnetic fields, which complicate the picture even more. There is also the question of the distance of water molecules from the sites of the ions and the influence of tunnelling effects. At present we attribute the major effect to outer sphere relaxation, involving a significant Curie contribution.

Regarding $r_{2}$, a similar behaviour is observed for all the different clusters: It is interesting to note that the $\mathrm{Fe}_{10} \mathrm{Dy}_{10}$ cluster shows the highest transverse relaxivity, whereas $\mathrm{Fe}_{10} \mathrm{Gd}_{10}$ exhibits the smallest. But all of them increase monotonically with increasing field. At the highest fields, large 
relaxivities are observed which is equivalent with the possibility of detecting rather small amounts of the relaxation agents due to the shortening of the transverse relaxation time.

From the perspective of MRT, $\mathrm{Fe}_{10} \mathrm{Gd}_{10}$ seems at a first sight the most interesting cluster as the high $r_{1}$ leads to a signal enhancement when measuring at high repetition rates in a non-equilibrium state of magnetization. $r_{2}$ is still moderate also at the fields currently used in material science. This means that the echo times can still be chosen such that a reasonably good spatial resolution is obtainable. To give some experimentally relevant numbers: $T_{2}$ relaxation time of water is in the order of $1 \mathrm{~s}$, a $1 \mathrm{mM} \mathrm{Fe}{ }_{10} \mathrm{Gd}_{10}$ solution will exhibit a transverse relaxation time around $28.6 \mathrm{~ms}$ at a ${ }^{1} \mathrm{H}$ Larmor frequency of $200 \mathrm{MHz}$. When imaging a sample by a multi slice multi echo sequence, spin echo times are usually in the order of several ms. Pure water is therefore hardly attenuated. In the case of the $1 \mathrm{mM} \mathrm{Fe}_{10} \mathrm{Gd}_{10}$ solution, the signal will be attenuated already to roughly $3 / 4$. On the other hand, $T_{1}$ is shortened from around $2-3 \mathrm{~s}$ to $42 \mathrm{~ms}$. On the time scale of the recovery time of an imaging experiment, the magnetization is fully relaxed leading to a drastic signal enhancement compared to pure water.

The simulation (Fig. 3) illustrates the situation for three concentrations of $\mathrm{Fe}_{10} \mathrm{Gd}_{10}$ in a range relevant for applications. The relative signal amplitude of water is calculated for different cluster concentrations as a function of time for both the transverse magnetization decay and the longitudinal recovery. The typical ranges of the MRT parameters used in the study on biofilms are marked. The conclusion from these graphs is that the discrimination of "pure" water is mostly due to the longitudinal contrast. The discrimination of different $\mathrm{Fe}_{10} \mathrm{Gd}_{10}$ concentrations, on the other hand, is achieved more easily in transverse magnetization. This fact has to be considered when choosing a cluster for MRT investigations: Since the $\mathrm{Fe}_{10} \mathrm{Dy}_{10}$ cluster shows the largest $r_{2}$ value and as the sensitivity to concentration is therefore the highest, this cluster is also of interest as a relaxation agent and will be examined in further studies. An additional point should be mentioned: Here we make the assumption that the order of magnitudes of relaxivities is more or less independent of the environment. This assumption has to be checked, as PRE strongly depends on molecular arrangements and tumbling frequencies. ${ }^{20-22}$

During the measurements, some samples started to separate especially at the lower concentrations up to $0.30 \mathrm{mM}$ after about 24 hours. This phenomenon of separation had, of course, a large influence on the NMR relaxation times, and multi-exponential recovery curves were detected. In Fig. 4 a
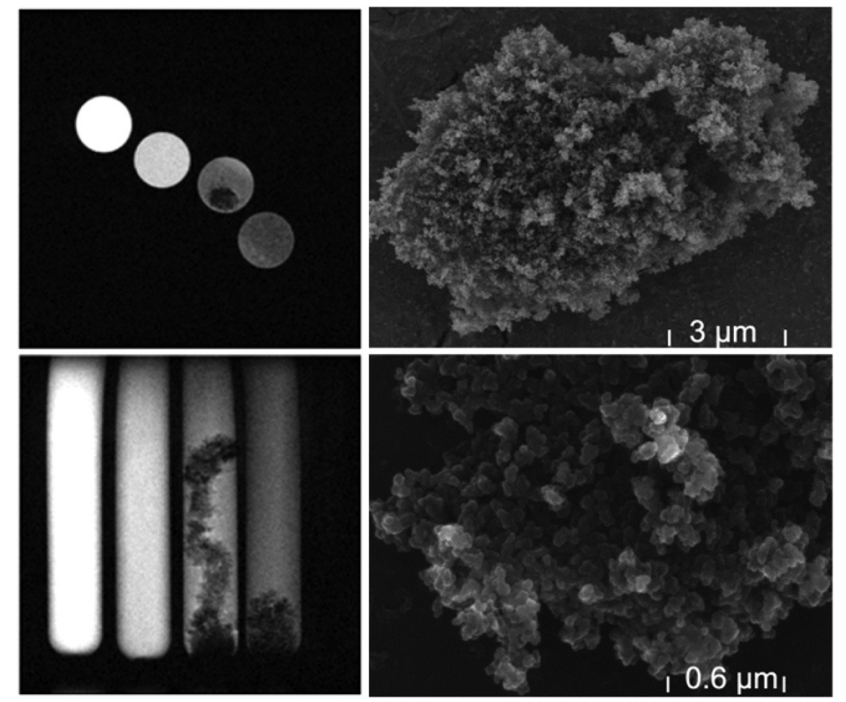

Fig. 4 The trend of the clusters to agglomerate is visible directly in MRT images (axial and sagittal slices on the left). Some structured agglomerates are formed which exhibit extremely fast transverse relaxations and therefore appear black in the image. Additional TEM images (right) show the fissured surface of the agglomerates leading to a large effective surface. This structure still makes PRE effective in the near neighbourhood.
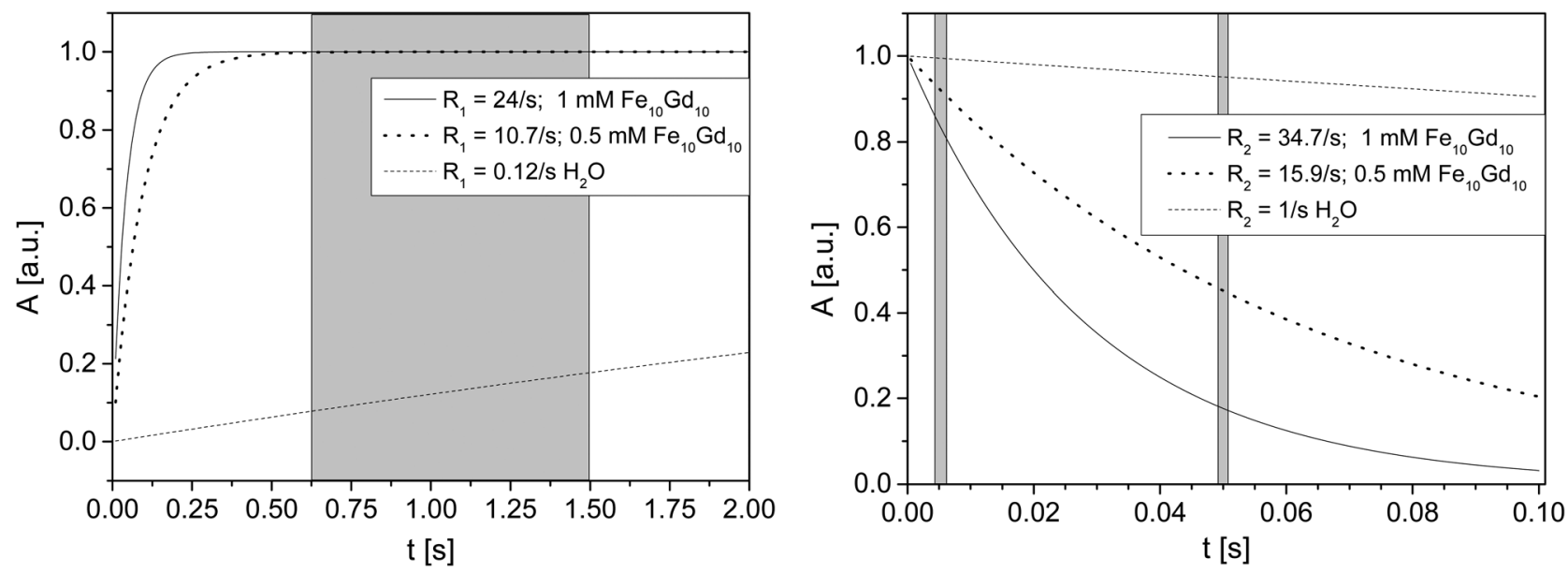

Fig. 3 Longitudinal ${ }^{1} \mathrm{H}$-magnetization build-up of water molecules (left) in the presence of different concentrations of $\mathrm{Fe}_{10} \mathrm{Gd}_{10}$ at $200 \mathrm{MHz}$ : "pure" water can clearly be discriminated due to the orders of magnitude different longitudinal relaxation. Typical experimental values are indicated by the grey shaded areas. On the right, the transverse magnetization decay is shown, again with the typically applied values of echo times. In this case, the concentration differentiation is substantial within the chosen experimental window. 
typical MRT image of the aggregation phenomenon is shown together with TEM images which reveal the surface structure of the agglomerates. In the MRT images, different concentrations were investigated from $1 \mathrm{mM}$ to $0.1 \mathrm{mM}$, the lower concentrations show the agglomerates. To avoid problems with this trend of aggregation or agglomeration of the clusters, samples were prepared immediately before running the NMR experiments, and special care was taken that no aggregation processes occurred during the relaxivity measurements. Due to the inhomogeneous structure of biofilms, which are presented in the next section, this effect may also occur in the biofilmcarrier systems and probably reinforces the observed contrast, since image contrast is enhanced with respect to inhomogeneities and interfaces, in turn facilitating the observation of details in the water-rich biofilms.

\section{Imaging of biofilms: transport and penetration of small molecules into the biofilm matrix}

Biofilms are formed by attachment of microorganisms to interfaces and are omnipresent in natural as well as in technical systems. ${ }^{23}$ They form various structures with smooth, rough, fluffy, porous or compact texture which influence the biological activity. ${ }^{24}$ In municipal waste water systems, biofilms on carrier material (Fig. 5) are a popular option for biological treatment. ${ }^{25}$ Transport of substrates to the biofilm surface is essential for high turnover rates. The predominant transport process in the concentration layer at the interface bulk/biofilm and in the biofilm itself is diffusion. ${ }^{26}$ Advection dominates the transport in the surrounding. For a better understanding of structure and functionality, MRT contrast agents are used for structure determination and as tracers for mass transport. Recent studies show that the spatially and time resolved distribution of contrast agents like Gd-chelates can help to noninvasively determine effective diffusion coefficients of model systems such as agar and pure culture biofilms. ${ }^{27,28}$ The present systems are more complex: The heterogeneous biofilms grown on irregularly shaped carrier material have open patchy structures and fluffy surfaces.

As little is known about the details of molecular transport into this technical biofilm matrix, the approach in the present paper is to apply different contrast agents with different mole-
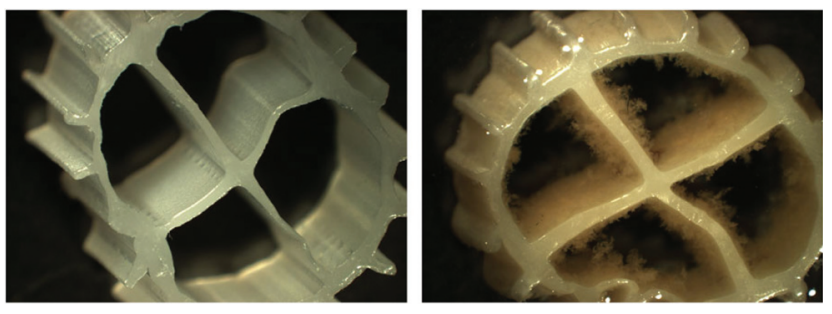

Fig. 5 Biofilm cultivated on carrier material: on the left, a stereomicroscope image of the blank plastic carrier is shown, which is divided into four sections. It has a diameter of $9 \mathrm{~mm}$ and a height of $7 \mathrm{~mm}$. On the right, a carrier is depicted on which biofilm has grown. Depending on growth conditions and nature of the biofilm community, the structure of the films is different, exhibiting also different mass transport properties. cular sizes as well as with different chemo-physical adsorption properties. With 4-Hydroxy-TEMPO, a small and hydrophilic molecule, a contrast agent is available, which does not agglomerate nor shows a pronounced surface activity (images not shown). Conventional Gadovist was applied, being well known as a medical relaxation agent, which can pass through tissue. The axial images obtained from long-term experiments after the injection of a bolus are compared with those obtained when applying the mentioned $\mathrm{Fe}_{10} \mathrm{Gd}_{10}$ clusters. The slice thickness was chosen to be $0.7 \mathrm{~mm}$, the spatial resolution in the image plane is $80 \mu \mathrm{m} \times 80 \mu \mathrm{m}$, acquiring 256 data points in each direction for $T_{1}$ weighted images. For $T_{2}$ weighted and proton density images, a resolution of $160 \mu \mathrm{m} \times 160 \mu \mathrm{m}$, acquiring 128 data points in each direction, was chosen.

A carrier with biofilm has a relatively homogenous proton density distribution (Fig. 6), which is measured also with a multi slice multi echo sequence, but with the smallest possible echo time of $3.2 \mathrm{~ms}$ ( $4.5 \mathrm{~ms}$ for $T_{1}$ weighted images) and a repetition time of $10 \mathrm{~s}$ to minimize the influence of relaxation contributions. Only the carrier itself can be clearly differentiated as the transverse relaxation is very short for hard polymer materials. The biofilm has an open structure and a low biomass density. The molecular mobility is not sufficiently hindered to show up in the relaxation times and surface related relaxation effects are found not to be dominant in the present system. In the middle of the upper row, a $T_{1}$ weighted image allows the differentiation of biofilm and surrounding water in the bulk at short repetition times (in our case $800 \mathrm{~ms}$ ) as "pure" water $T_{1}$ relaxation is much slower than $T_{1}$ of water in the biofilm. Finally, the $T_{2}$ weighted image shows the reverse contrast: Biofilm appears with lower amplitudes compared with the surrounding water as $T_{2}$ in the biofilm is shorter, which is in accordance with ref. 29. In the row in the middle of Fig. 6, $T_{1}$ weighted images are displayed: In a reference image shown on the left the biofilm is barely visible on the carrier. When injecting Gadovist and waiting $58 \mathrm{~h}$, the sections of the carrier, in which Gadovist was injected, appear with higher amplitudes, but still show little contrast to the water in the sections. When replacing the water in the carrier with fresh water and acquiring a $T_{1}$ weighted image afterwards (right), regions of water and of biofilm can be differentiated. Water with the larger $T_{1}$ appears in dark grey within the section. The picture becomes clearer in the case of $T_{2}$ weighted images of the same carrier (bottom row), again on the left the reference image without Gadovist is given. In the middle, the image shows the biofilm directly after injection, whereas $59 \mathrm{~h}$ passed before acquiring the image on the right.

These experiments clearly show the influence of the relaxation agent. The $T_{2}$ weighted images were recorded directly after the $T_{1}$ weighted images to reflect the same state of the biofilm. In the $T_{2}$ weighted images acquired after injection, the adsorption of the contrast agent into the biofilm surface is clearly visible. It seems that the fluffy biomass accumulated most of the Gadovist near the surface due to the high surface contact area. By comparing these to the images taken when no contrast agent was added, the full penetration of the Gadovist 

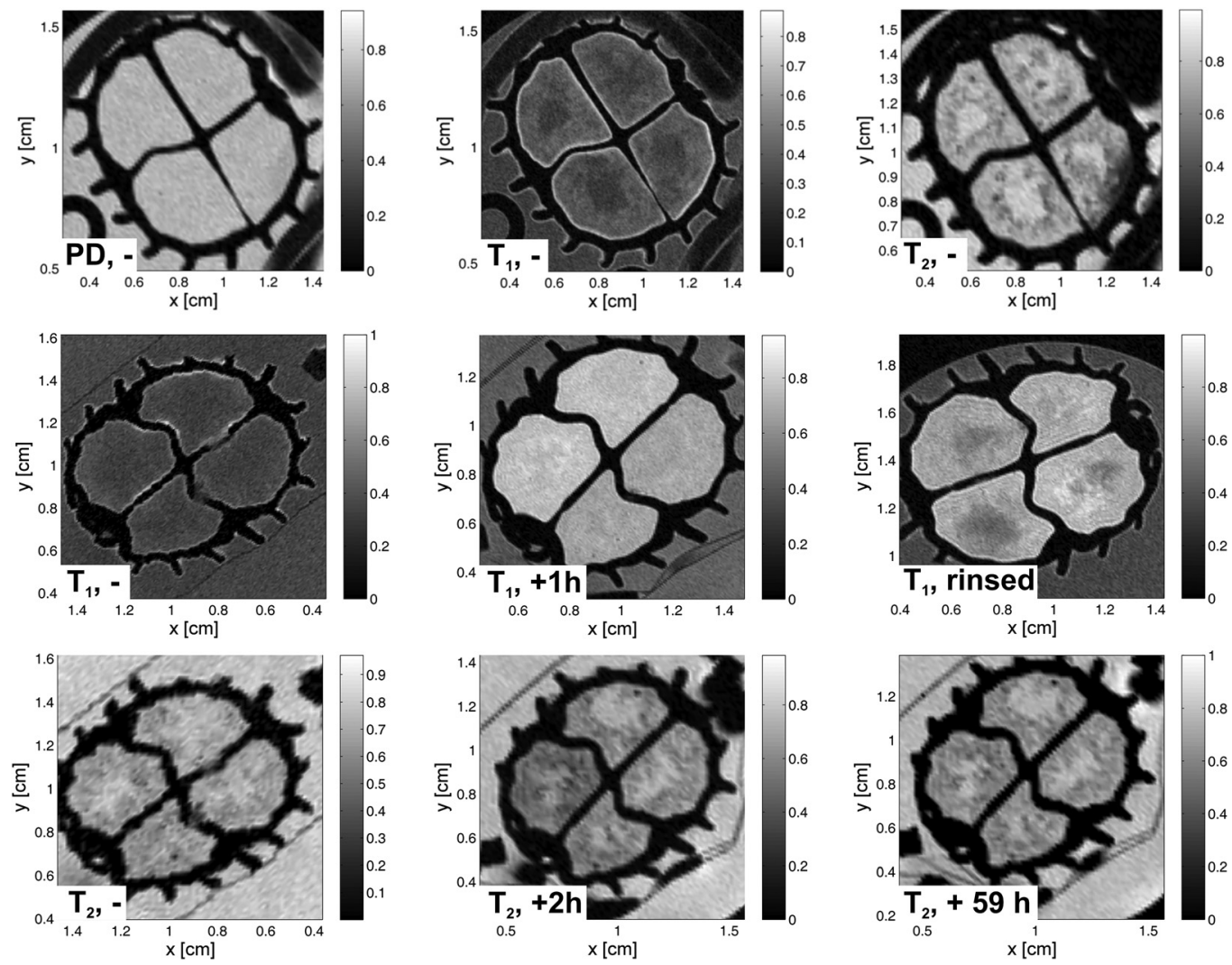

Fig. 6 MRT axial slices in the middle of the carrier as measured by applying a multi-slice multi-echo sequence. In the upper row, images with different MRT parameters are shown, which lead to quite different image contrast (from left to right): proton density (PD), $T_{1}$ and $T_{2}$ weighted images of a carrier with biofilm, but without any contrast agent. The row in the middle displays images of an experiment, where Gadovist was added: on the left, a $T_{1}$ weighted reference image without Gadovist, in the middle the corresponding image after $1 \mathrm{~h}$ of injection, and on the right a $T_{1}$ weighted image after rinsing the carrier with tap water showing the remaining Gadovist in the biofilm matrix. In the lower row, the corresponding $T_{2}$ weighted images of the same experiment are shown, starting from the reference image, the image directly after injection and ending with the image after $59 \mathrm{~h}$ of exposure to Gadovist.

into the biomass is underlined. After rinsing the biofilm carriers the Gadovist situated in the bulk medium was removed. This indicates that Gadovist diffuses into the biofilm, and a dynamic equilibrium is observed between the water phase and the biofilm.

A similar experiment was performed injecting $\mathrm{Fe}_{10} \mathrm{Gd}_{10}$ as contrast agent into the sections of the carrier. In Fig. 7, the corresponding MRT images are shown as axial slices with a thickness of $0.7 \mathrm{~mm}$ and the same in-plane resolution as stated above. The rows correspond to $T_{1}$ weighted images and $T_{2}$ weighted (proton density) images, respectively. The left column is again the reference, the lower one being a $T_{2}$ weighted image with an echo time of $50 \mathrm{~ms}$. The contrast between water and biofilm is relatively poor. Directly after injection, images were acquired which show the influence of the high-spin cluster due to PRE as discussed in context of the relaxivity study. Interestingly, the surfaces of the biofilm become clear in both the $T_{1}$ weighted and the proton density images, respectively. Structural features can be identified in the $T_{1}$ weighted images, probably arising from the tendency of the $\mathrm{Fe}_{10} \mathrm{Ln}_{10}$ clusters to agglomerate. Given that the interfaces of biofilms are usually hard to detect, the $\mathrm{Fe}_{10} \mathrm{Gd}_{10}$ used here provides a novel and selective contrast enhancement. Furthermore, it bridges the gap between molecular contrast agents and classical magnetic nanoparticles.

After $18 \mathrm{~h}, T_{1}$ weighted and proton density images with identical MRT parameters were recorded; this showed that the situation did not change significantly. As shown in the section "Experimental", the clusters tend to agglomerate, therefore the created contrast after injection of $\mathrm{Fe}_{10} \mathrm{Gd}_{10}$ can be understood as being due to the presence of the clusters especially on the biofilm surfaces. A quite strong interaction seems to be present, as no signature of diffusion neither into the surrounding water nor into the biofilm is obvious in the images. Furthermore, after stirring the solution, the effect increases (Fig. 7, right column): The $T_{2}$ weighted image (Fig. 7, bottom row) shows this process very clearly. The short transverse relaxation times lead to vanishing signal amplitudes in the neighbourhood of the clusters and to a strong negative contrast in the image. Since the longitudinal relaxivity is also relatively large, the positive contrast is still visible in the $T_{1}$ weighted image when using the smallest possible echo time. The comparison of both parameterized images of the same object in a similar state allows therefore the localization of the clusters at 

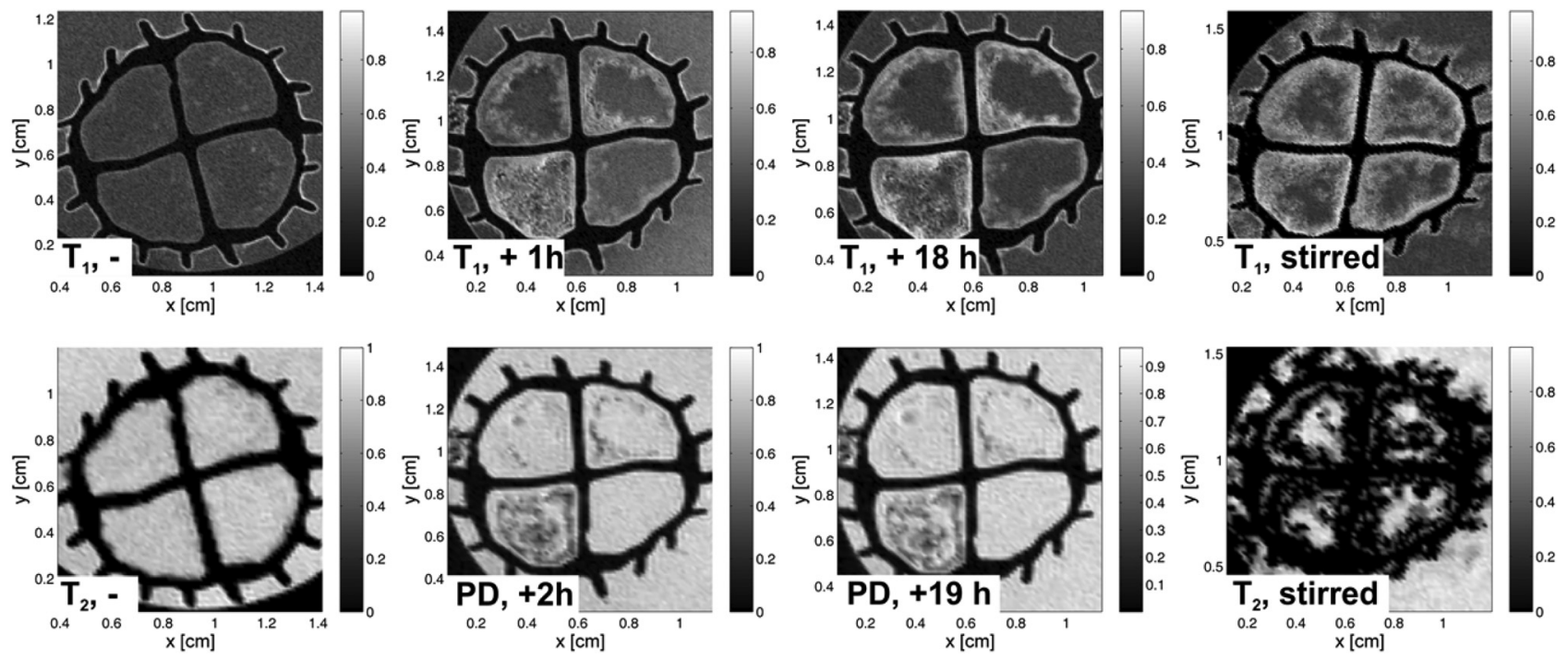

Fig. $7 T_{1}$ weighted, $T_{2}$ weighted and proton density (PD) images of a biofilm carrier, which was exposed to $\mathrm{Fe}_{10} \mathrm{Gd}_{10}$. For comparison, reference images are shown on the left $\left(T_{1}\right.$ and $T_{2}$ weighted, respectively). After injection, both, $T_{1}$ and proton density images show the presence of Fe ${ }_{10} \mathrm{Gd}_{10}$, interestingly the surface of the biofilm is marked very pronounced. After $18 \mathrm{~h}$, the situation did not change significantly, which leads to the conclusion that the $\mathrm{Fe}_{10} \mathrm{Gd}_{10}$ clusters attach to the surfaces and do not significantly migrate into the biofilm further on. After stirring the vessel, the effect becomes even more pronounced (right most column: $T_{1}$ and $T_{2}$ weighted images).

the biofilm's interfaces. When observing $\mathrm{Fe}_{10} \mathrm{Gd}_{10}$ as contrast agent in the carriers and when comparing with the behaviour of Gadovist it can be concluded that here penetration occurs more slowly than that of Gadovist, probably due to the molecular structure (mass, shape, surface functionality and radius: about $1.4 \mathrm{~nm}$ compared to a hydrodynamic radius of $0.9 \mathrm{~nm}$ for Gadovist) and especially due to agglomeration processes. In contrast to Gadovist, where a relatively unspecific reaction is observed, the $\mathrm{Fe}_{10} \mathrm{Gd}_{10}$ cluster focuses the image contrast onto the biofilm interfaces, seen especially clearly in the proton density and in the $T_{2}$ weighted images due to the large $r_{2}$ value. Less contrast agent is found in the "pure" bulk. This shows that $\mathrm{Fe}_{10} \mathrm{Gd}_{10}$ has a certain affinity for the biofilm's interfaces. Furthermore, differences in the biofilm structure can be imaged by focusing on areas where the contrast agents could penetrate as a function of time. The application of the $\mathrm{Fe}_{10} \mathrm{Gd}_{10}$ to fluffy biofilms offers new possibilities to visualize the biofilm/bulk boundary as well as to study the behaviour of contrast agents in biological systems - provided the biological compatibility and toxicological issues are proven.

\section{Conclusion}

The relaxivities of the highly paramagnetic cooperative clusters $\mathrm{Fe}_{10} \mathrm{Ln}_{10}$ were measured over a wide range of ${ }^{1} \mathrm{H}$ Larmor frequencies from $10 \mathrm{MHz}$ up to $1.4 \mathrm{GHz}$. Both relaxivities, $r_{1}$ and $r_{2}$, depend on the nature of $\mathrm{Ln}^{\mathrm{III}}$. The transverse relaxivity $r_{2}$ increases monotonically with field for all the investigated paramagnetic $\mathrm{Ln}{ }^{\mathrm{III}}$ ions as does the longitudinal relaxivity $r_{1}$ except for that of $\mathrm{Fe}_{10} \mathrm{Gd}_{10}$, which is almost constant up to high Larmor frequencies. As far as the clusters are presently under- stood, the major contributions to PRE are due to outer sphere dipolar and Curie contributions.

In the first MRT application of $\mathrm{Fe}_{10} \mathrm{Gd}_{10}$ in a study of biofilms from waste water treatment, the PRE effect is confirmed as expected from the relaxivity investigations. Furthermore, a pronounced adhesion is observed on the biofilm's surfaces. As the clusters preferably adhere to the fractional surface of the biofilm, the image contrast is modified such that interfaces can be investigated in more detail.

\section{Acknowledgements}

Our work was funded by the German State of Baden-Württemberg, the Karlsruhe Institute of Technology (KIT) and the Deutsche Forschungsgemeinschaft as administrated through the Center for Functional Nanostructures (CFN) and within the DFG-instrumental facility center Pro ${ }^{2}$ NMR. LNCMI Grenoble is member of the European Magnetic Field Laboratory (EMFL), and part of this work there has been supported by EuroMagNET under the EU contract no. 228043. Financial support of Julyana R. Machado was provided by Csf/CNPq-Conselho Nacional de Desenvolvimento Científico e Tecnológico, of the Ministry of Science, Technology and Innovation from Brazil, which is kindly acknowledged. Maria P. Herrling thanks the German Carl-Zeiss Foundation for financial support. Special thanks to Dr Jochen Junker for helpful discussions, to Dr Mladen Horvatić and Dr Hadrien Mayaffre for assistance at the LNCMI, and to Vanessa Grande for laboratory support. The relaxivities of water in the presence of $\mathrm{Fe}_{10} \mathrm{Lu}_{10}$ were investigated by $\mathrm{Y}$. Maier. The TEM images and size analysis were performed at MVM by A. Huber, K. Hirsch, and T. Lebe, sincere thanks are given to them all. 


\section{Notes and references}

1 A. Baniodeh, PhD-Thesis, Karlsruher Institut für Technologie (KIT), 2012.

2 A. Baniodeh, C. E. Anson and A. K. Powell, Chem. Sci., 2013, 4, 4325-4536.

3 J. R. Machado, A. Baniodeh, A. K. Powell, B. Luy, S. Krämer and G. Guthausen, ChemPhysChem, 2014, 15, 36083613.

4 X. P. Yang, D. Schipper, R. A. Jones, L. A. Lytwak, B. J. Holliday and S. M. Huang, J. Am. Chem. Soc., 2013, 135, 8468-8471.

5 I. Bertini, C. Luchinat and G. Parigi, Solution NMR of paramagnetic molecules: Applications to metallobiomolecules and models, Elsevier Science B.V., Amsterdam, 1st edn, 2001.

6 A. Baniodeh, Y. Liang, C. E. Anson, N. Magnani, A. K. Powell, A.-N. Unterreiner, S. Seyfferle, M. Slota, M. Dressel, L. Bogani and K. Goß, Adv. Funct. Mater., 2014, 24, 6280-6290.

7 M. Bottrill, L. K. Nicholas and N. J. Long, Chem. Soc. Rev., 2006, 35, 557-571.

8 P. H. Fries, J. Chem. Phys., 2012, 136, 044504-044504.

9 Y. Gossuin, A. Roch, F. Lo Bue, R. N. Muller and P. Gillis, Magn. Reson. Med., 2001, 46, 476-481.

10 D. Kruk, A. Korpala, J. Kowalewski, E. A. Rössler and J. Moscicki, J. Chem. Phys., 2012, 137, 044512-044512.

11 Q. L. Vuong, S. Van Doorslaer, J. L. Bridot, C. Argante, G. Alejandro, R. Hermann, S. Disch, C. Mattea, S. Stapf and Y. Gossuin, Magn. Reson. Mater. Phys., 2012, 25, 467-478.

12 S. Laurent, L. Vander Elst and R. N. Muller, Contrast Media Mol. Imaging, 2006, 1, 128-137.

13 I. Bertini, F. Capozzi, C. Luchinat, G. Nicastro and Z. C. Xia, J. Phys. Chem., 1993, 97, 6351-6354.
14 L. Van der Elst, A. Roch, P. Gillis, S. Laurent, F. Botteman, J. W. M. Bulte and R. N. Muller, Magn. Reson. Med., 2002, 47, 1121-1130.

15 T. Nilsson, G. Parigi and J. Kowalewski, J. Phys. Chem. A, 2002, 106, 4476-4488.

16 S. Laurent, L. Vander Elst, A. Roch and R. N. Muller, in NMR-MRI, $\mu S R$ and Mössbauer Spectroscopies in Molecular Magnets, ed. P. Carretta and A. Lascialfari, Springer, 2007, pp. 71-87.

17 G. Pintacuda, M. John, X. C. Su and G. Otting, Acc. Chem. Res., 2007, 40, 206-212.

18 A. Roch, Y. Gossuin, R. N. Muller and P. Gillis, J. Magn. Magn. Mater., 2005, 293, 532-539.

19 C. J. Meledandri, J. K. Stolarczyk, S. Ghosh and D. F. Brougham, Langmuir, 2008, 24, 14159-14165.

20 J. L. Barnhart, N. Kuhnert, D. A. Bakan and R. N. Berk, Magn. Reson. Imaging, 1987, 5, 221-231.

21 J. L. Barnhart and R. N. Berk, Invest. Radiol., 1986, 21, 132-136.

22 Y. Gossuin, C. Burtea, A. Monseux, G. Toubeau, A. Roch, R. N. Muller and P. Gillis, J. Magn. Reson. Imaging, 2004, 20, 690-696.

23 J. W. Costerton, Z. Lewandowski, D. E. Caldwell, D. R. Korber and H. M. Lappinscott, Annu. Rev. Microbiol., 1995, 49, 711-745.

24 H.-C. Flemming and J. Wingender, Nat. Rev. Microbiol., 2010, 8, 623-633.

25 H. Odegaard, Water Sci. Technol., 2006, 53, 17-33.

26 P. S. Stewart, J. Bacteriol., 2003, 185, 1485-1491.

27 B. Ramanan, W. M. Holmes, W. T. Sloan and V. R. Phoenix, Appl. Environ. Microbiol., 2010, 76, 4027-4036.

28 B. Ramanan, W. M. Holmes, W. T. Sloan and V. R. Phoenix, Curr. Microbiol., 2013, 66, 456-461.

29 B. Manz, F. Volke, D. Goll and H. Horn, Biotechnol. Bioeng., 2003, 84, 424-432. 